\title{
Review Article \\ Modeling of Stacking Fault Energy in Hexagonal-Close-Packed Metals
}

\section{Zhigang Ding, Shuang Li, Wei Liu, and Yonghao Zhao}

Nano Structural Materials Center, School of Materials Science and Engineering, Nanjing University of Science and Technology, Nanjing, Jiangsu 210094, China

Correspondence should be addressed to Wei Liu; weiliu@njust.edu.cn and Yonghao Zhao; yhzhao@njust.edu.cn

Received 19 January 2015; Accepted 20 April 2015

Academic Editor: Filippo Giannazzo

Copyright (C) 2015 Zhigang Ding et al. This is an open access article distributed under the Creative Commons Attribution License, which permits unrestricted use, distribution, and reproduction in any medium, provided the original work is properly cited.

The deformation of metals is known to be largely affected by their stacking fault energies (SFEs). In the review, we examine the theoretical background of three normally used models, supercell model, Ising model, and bond orientation model, for the calculation of SFE of hexagonal-close-packed (hcp) metals and their alloys. To predict the nature of slip in nanocrystalline metals, we further review the generalized stacking fault (GSF) energy curves in hcp metals and alloys. We conclude by discussing the outstanding challenges in the modeling of SFE and GSF energy for studying the mechanical properties of metals.

\section{Introduction}

Due to the high fracture toughness, uniform property in all directions, conductivity, and recyclability, metals are dominant in engineering applications. Strength and ductility are two important mechanical properties for metal structure materials; however, it is difficult for a material to have both high strength and high ductility [1]. For face-centered cubic (fcc) metals, such as $\mathrm{Cu}$ alloys, deformation twinning is a useful mechanism that can simultaneously enhance the strength and ductility $[2,3]$. The factors affecting the deformation twinning include strain rate [4], deformation temperature [5], grain size [6], and stacking fault energy (SFE) $[7,8]$. In contrast, the hexagonal-close-packed (hcp) metals usually have low plasticity due to limited independent slip systems. In hcp systems, Kwaśniak et al. [9] revealed that the interplanar distance of prismatic and pyramidal slip planes is different. They listed the possible slip system of hcp metals as $\langle a\rangle$ basal, $\langle a\rangle$ prismatic I, $\langle a\rangle$ prismatic II, $\langle a\rangle$ pyramidal I, $\langle a\rangle$ pyramidal II, $\langle c+a\rangle$ pyramidal I, and $\langle c+a\rangle$ pyramidal II, where I and II denote large or small interplanar distance. This dual nature of pyramidal and prismatic planes has not been reported in experimental or theoretical studies.

SFE is commonly accepted as a useful quantity for understanding the plastic deformation mechanisms of metals. Deformation twinning is favored at low SFE and dislocation slip at high SFE. In high-manganese austenitic steels, for instance, the mechanical twinning is for low values, while dislocation slip is for high values of the SFE [10]. The introduction of additional alloy elements is also an effective approach to improve the mechanical properties of metals. By adding alloy atoms, the SFE of $\mathrm{Cu}-\mathrm{Zn}$ alloy has been readily adjusted, producing high strength and high ductility. Suzuki et al. [11] claimed that, with the addition of $\mathrm{Zn}$, the creep strength of $\mathrm{Mg}-\mathrm{Y}$ alloys improved at $550-650 \mathrm{~K}$ and decreased the SFE values. In addition, Kawamura et al. [12] synthesized $\mathrm{Mg}_{97} \mathrm{Y}_{2} \mathrm{Zn}_{1}$ alloys by a rapidly solidified powder metallurgy technique and achieved a high tensile strength of $600 \mathrm{MPa}$ and elongation of 5\%, along with a decrease of the SFE value.

However, the effect of solute atoms on SFE remains unclear, which is mainly because the magnitudes of SFE are small, being difficult for experimental measurement. Accordingly, density-functional theory (DFT) becomes an effective and popular method to calculate the SFEs for metal surfaces.

In this review, we will focus on the modeling of the SFE in hcp metals. In Section 2, we will briefly introduce the definition of stacking fault and generalized stacking fault (GSF) energy. The models used for first principle calculations will be elucidated in Section 3. In Section 4, we will compare 
the GSF energies determined by different DFT functional. The challenge and outlook for the modeling methods will be shown in Section 5.

\section{Stacking Fault Energy and Generalized Stacking Fault Energy}

On the microscale, atoms were described as hard spheres which change size from one element to another. Perfect crystals can be described by the hard spheres stacking at three-dimensional space in a regular sequence. The atoms in one layer are identical; the sequence of atom layers in the crystal is described by labeling one layer and other layers with atoms in identical positions as an A layer; layers of atoms in other positions in the stack are referred to as B or C layers. Stacking faults, which appear at the close-packed planes in the close-packed structures (fcc and hcp), describe the change of local region in crystals by removal or introducing external layers. Stacking faults destroy the perfection of the ideal structure, and the accompanied energy per unit area of faults is known as SFE $\gamma$ [13].

According to the hard-sphere models of atoms, four basal plane faults exist compared to the perfect sequence ... ABABAB .... Two are intrinsic and called $I_{1}$ and $I_{2}$. Fault $I_{1}$, or growth fault, is formed by removal of a basal plane, followed by slip of the crystal above this fault of $(1 / 3)\langle 10 \overline{1} 0\rangle$ :

$$
\begin{aligned}
\ldots \mathrm{ABABABAB} \ldots & \longrightarrow \ldots \text { АВАВВАВА } \ldots \\
& \longrightarrow \ldots \text { АВАВСВСВ } \ldots
\end{aligned}
$$

Fault $I_{2}$, or deformation fault, formed from slip of (1/ $3)\langle 10 \overline{1} 0\rangle$ in a perfect crystal:

$$
\ldots \text { ABABABAB } \ldots \rightarrow \ldots \text { ABABCACA .... }
$$

The external fault $E$ is formed by inserting an extra plane:

$$
\ldots \text { ABABABAB } \ldots \rightarrow \ldots \text { ABABCABAB .... }
$$

The twin-like fault $T_{2}$ has mirror symmetry structure about the fault plane:

$$
\ldots \text { ABABABAB } \ldots \rightarrow \text { ABABCBAB } \ldots .
$$

With the model described above, the energy of an atom in the system is determined by the local environment (layers close to it). In perfect crystals, hcp structures have a local hcp-like environment and fcc structures have a local fcclike environment. The main contribution to $\gamma$ arises from the changes of the local environment of atoms. For example, in the ideal hcp structure, the stacking sequence is ... ABABAB ..., but, in the faulted sequences, some planes have a local fcc-like environment. There are one fcc-like atom in $I_{1}$, two in $I_{2}$ and $T_{2}$, and three in $E$; then the SFE satisfies $\gamma_{E} \approx(3 / 2) \gamma_{I_{2}} \approx(3 / 2) \gamma_{T_{2}} \approx 3 \gamma_{I_{1}}$ [13]. Experimental estimates of $\gamma$ often show large uncertainties and are different from the theoretical estimate. For instance, the SFEs measured for magnesium are reported to range from $50 \mathrm{~mJ} / \mathrm{m}^{2}$ to $280 \mathrm{~mJ} / \mathrm{m}^{2}$ [14]. Fortunately, the DFT calculations could give reliable estimates of the SFEs at reasonable computer cost. For hcp metals, the basal close-packed plane is (0001) plane and the close-packed direction is $\langle 11 \overline{2} 0\rangle$. Along this direction, the dislocation glide is frequently observed. $\mathrm{Mg}$ has a $c / a$ ratio of 1.632 , which is close to ideal close-packed atomic spheres. Its dislocation can also easily slip along the $(1 / 3)\langle 11 \overline{2} 0\rangle$ direction on the first-order prism planes $\{10 \overline{1} 0\}$ and pyramidal plane $\{10 \overline{1} 1\}[13]$.

Since the stacking fault described above only locates at stable sites, it is thus named "stable SFE". In contrast, the SFE related to the slip process is referred to as the "unstable SFE". When studying the deformation properties or dislocation core, it is important to export the evolution of SFEs from one stable position to another. The generalized stacking fault (GSF) energy surface ( $\gamma$-surface) can provide a description of stacking faults evolution. The GSF energy was first introduced by Vítek, for the description of the dislocation core structure and mobility [15]. The GSF energy is described by the difference of two supercells, which can be used to model stacking faults with the burger vectors $b=0$ (stable position) and $b \neq 0$ (unstable position).

\section{Theoretical Models for Stacking Fault Energy}

For SFE calculations, there are three typical models: (1) supercell model, (2) Ising model, and (3) bond orientation model. The most commonly used supercell model based on the geometry structure will be shown in Section 3.1. The other two models based on different parameters will be shown in Sections 3.2 and 3.3.

3.1. Supercell Model. In 1980s, Chou et al. [16] placed stacking faults in a periodic repeated supercell, where two large supercells are required for SFE calculations. One is the structure of perfect crystal; the other is the structure containing stacking fault; calculate the energy of the two structures by first principle calculations directly. Then, the definition of SFE $\gamma$ can be expressed as

$$
\gamma=\frac{E_{\mathrm{SF}}-E_{0}}{A}
$$

where $E_{\mathrm{SF}}$ denotes the energy of stacking fault structure, $E_{0}$ the energy of perfect structure, and $A$ the surface area.

Based on this model, four basal plane SFEs were calculated by Chetty and Weinert using LDA [17] and by Smith using GGA [18] (cf. Table 1). The $I_{1}$ and $I_{2}$ SFEs are found to be the most stable and to have a lower energy than the ideal fcc Mg. However, the results of SFE are closely related to the size of the supercell. For example, Wang et al. [19] revealed that when the supercell size increases from 8 to 32 , the SFE reduced by $50 \%$. Recently, several long-period stacking ordered (LPSO) structures have been found in Mg$\mathrm{Y}$ alloys; these alloys show superplastic behavior at high temperatures $[20,21]$. First principle calculations were used to investigate the LPSO structures and showed that the SFEs of these structures only contain $I_{1}$ and $I_{2}$ type stacking fault [22]. The SFEs of other hcp metals (Be, Sc, Y, Ti, Zr, Tc, Re, 
TABLE 1: Calculated stable SFE $\left(\mathrm{mJ} / \mathrm{m}^{2}\right)$ and unstable SFE $\left(\mathrm{mJ} / \mathrm{m}^{2}\right)$ for $\mathrm{Mg}$ and $\mathrm{Mg}$ alloys by DFT with different exchange-correlation functionals.

\begin{tabular}{|c|c|c|c|c|c|c|c|c|}
\hline \multirow{2}{*}{ System } & \multicolumn{2}{|c|}{ Growth fault $\left(I_{1}\right)$} & \multicolumn{2}{|c|}{ Deformation fault $\left(I_{2}\right)$} & \multicolumn{2}{|c|}{ Extrinsic fault $(E)$} & \multicolumn{2}{|c|}{ Twin-like fault $\left(T_{2}\right)$} \\
\hline & Stable SFE & Unstable SFE & Stable SFE & Unstable SFE & Stable SFE & Unstable SFE & Stable SFE & Unstable SFE \\
\hline $\mathrm{Mg}$ & $\begin{array}{c}21^{\mathrm{a}}, 18^{\mathrm{b}}, 16^{\mathrm{c}}, 30^{\mathrm{e}} \\
17.98^{\mathrm{f}}, 20^{\mathrm{g}}, 17.1^{\mathrm{h}} \\
\quad 17.8^{\mathrm{i}}, 18^{\mathrm{k}}\end{array}$ & $86.2^{\mathrm{h}}$ & $\begin{array}{c}44^{\mathrm{a}}, 36^{\mathrm{b}}, 34^{\mathrm{c}}, \\
36^{\mathrm{d}}, 60^{\mathrm{e}} \\
33.84^{\mathrm{f}}, 33.8^{\mathrm{h}} \\
38.3^{\mathrm{i}}, 33^{\mathrm{k}}\end{array}$ & $97^{\mathrm{c}}, 92^{\mathrm{d}}, 87.6^{\mathrm{h}}$ & $\begin{array}{c}69^{\mathrm{a}}, 58^{\mathrm{b}}, 59^{\mathrm{c}} \\
90^{\mathrm{e}}, 52.57^{\mathrm{f}}\end{array}$ & & $\begin{array}{c}51^{\mathrm{a}}, 40^{\mathrm{b}}, 38^{\mathrm{c}} \\
39^{\mathrm{d}}, 60^{\mathrm{e}} \\
40.55^{\mathrm{f}}\end{array}$ & $181^{\mathrm{c}}, 111^{\mathrm{d}}$ \\
\hline $\mathrm{Mg}-\mathrm{Li}$ & $16.7^{\mathrm{j}}, 28^{\mathrm{k}}$ & & $47^{\mathrm{d}}, 48.2^{\mathrm{j}}, 46^{\mathrm{k}}$ & $95^{\mathrm{d}}$ & & & $56^{\mathrm{d}}, 63.5^{\mathrm{j}}$ & $119^{\mathrm{d}}$ \\
\hline $\mathrm{Mg}-\mathrm{Na}$ & $15.7^{j}$ & & $46.8^{j}$ & & $66.7^{j}$ & & & \\
\hline $\mathrm{Mg}-\mathrm{Al}$ & $12.5^{j}$ & & $21^{\mathrm{d}}, 33.6^{\mathrm{j}}$ & $78^{\mathrm{d}}$ & $55.7^{j}$ & & $32^{\mathrm{d}}$ & $96^{\mathrm{d}}$ \\
\hline $\mathrm{Mg}-\mathrm{K}$ & $13.5^{j}$ & & $32.5^{j}$ & & $47.5^{j}$ & & & \\
\hline $\mathrm{Mg}-\mathrm{Ca}$ & $13.8^{j}$ & & $33.5^{j}$ & & $49.6^{j}$ & & & \\
\hline $\mathrm{Mg}-\mathrm{Ti}$ & & & $36^{\mathrm{d}}$ & $112^{\mathrm{d}}$ & & & $47^{\mathrm{d}}$ & $137^{\mathrm{d}}$ \\
\hline $\mathrm{Mg}-\mathrm{Mn}$ & $13.5^{j}$ & & $38^{\mathrm{d}}, 40.3^{\mathrm{j}}$ & $103^{\mathrm{d}}$ & $81.4^{\mathrm{j}}$ & & $83^{\mathrm{d}}$ & $149^{\mathrm{d}}$ \\
\hline $\mathrm{Mg}-\mathrm{Fe}$ & $3.3^{j}$ & & $52^{\mathrm{d}}, 40.3^{\mathrm{j}}$ & $110^{\mathrm{d}}$ & $79.8^{j}$ & & $80^{\mathrm{d}}$ & $146^{\mathrm{d}}$ \\
\hline $\mathrm{Mg}-\mathrm{Ni}$ & & & $55^{\mathrm{d}}$ & $99^{\mathrm{d}}$ & & & $81^{\mathrm{d}}$ & $135^{\mathrm{d}}$ \\
\hline $\mathrm{Mg}-\mathrm{Cu}$ & $16.6^{j}$ & & $53^{\mathrm{d}}, 40.1^{\mathrm{j}}$ & $97^{\mathrm{d}}$ & $66.4^{j}$ & & $68^{\mathrm{d}}$ & $126^{\mathrm{d}}$ \\
\hline $\mathrm{Mg}-\mathrm{Zn}$ & $18.2^{\mathrm{h}}, 8.5^{\mathrm{j}}$ & $91.0^{\mathrm{h}}$ & $\begin{array}{c}37^{\mathrm{d}}, 35.1^{\mathrm{h}}, \\
30.8^{\mathrm{j}}\end{array}$ & $86^{\mathrm{d}}, 94.2^{\mathrm{h}}$ & $58.8^{\mathrm{j}}$ & & $43^{\mathrm{d}}$ & $195^{\mathrm{d}}$ \\
\hline $\mathrm{Mg}-\mathrm{Sr}$ & $15.6^{\mathrm{j}}$ & & $33.2^{\mathrm{j}}$ & & $46.3^{j}$ & & & \\
\hline $\mathrm{Mg}-\mathrm{Y}$ & $3.7^{\mathrm{h}}, 1.8^{\mathrm{j}}$ & $61.2^{\mathrm{h}}$ & $\begin{array}{c}25^{\mathrm{d}}, 15.7^{\mathrm{h}}, \\
32.9^{\mathrm{j}}\end{array}$ & $71^{\mathrm{d}}, 62.1^{\mathrm{h}}$ & $47.1^{\mathrm{j}}$ & & $21^{\mathrm{d}}$ & $85^{\mathrm{d}}$ \\
\hline $\mathrm{Mg}-\mathrm{Zr}$ & $9.8^{j}$ & & $26^{\mathrm{d}}, 35.3^{\mathrm{j}}$ & $99^{\mathrm{d}}$ & $67.5^{j}$ & & $32^{\mathrm{d}}$ & $119^{\mathrm{d}}$ \\
\hline Mg-Ag & & & $49^{\mathrm{d}}$ & $101^{\mathrm{d}}$ & & & $62^{\mathrm{d}}$ & $127^{\mathrm{d}}$ \\
\hline $\mathrm{Mg}-\mathrm{Nd}$ & $10.8^{j}$ & & $32.3^{j}$ & & $51.9^{j}$ & & & \\
\hline $\mathrm{Mg}-\mathrm{Sn}$ & $13.8^{j}$ & & $2^{\mathrm{d}}, 37.2^{\mathrm{j}}$ & $64^{\mathrm{d}}$ & $50.1^{j}$ & & $25^{\mathrm{d}}$ & $79^{\mathrm{d}}$ \\
\hline $\mathrm{Mg}-\mathrm{Pb}$ & & & $4^{\mathrm{d}}$ & $55^{\mathrm{d}}$ & & & $32^{\mathrm{d}}$ & $80^{\mathrm{d}}$ \\
\hline
\end{tabular}

Note:

${ }^{a}$ Chetty and Weinert calculated with LDA [17].

${ }^{\mathrm{b}}$ Smith calculated with GGA-PBE [18].

${ }^{c}$ Wen et al. calculated with GGA-PW91 [28].

${ }^{\mathrm{d}}$ Muzyk et al. calculated with GGA-PBE; the concentration of alloying atoms was 2\% in supercell and 25\% in slip layers [29].

${ }^{\mathrm{e}} \mathrm{Hu}$ and Yang calculated with LDA [23].

${ }^{\mathrm{f}}$ Fan et al. calculated with GGA-PW91 [22].

${ }^{\mathrm{g}}$ Sandlöbes et al. calculated with GGA-PBE [20].

${ }^{\mathrm{h}}$ Zhang et al. calculated with GGA-PW91; the concentration of alloying atoms was $1 \%$ in supercell and $11.1 \%$ at the fault plane [32].

${ }^{\mathrm{i}}$ Wang et al. calculated with GGA [19].

${ }^{\mathrm{j}}$ Wang et al. calculated with GGA [35].

${ }^{\mathrm{k}} \mathrm{Han}$ et al. calculation with GGA of Mg-1.67\% Y alloys [30].

$\mathrm{Zn}, \mathrm{Cd}$, etc. $[23,24])$ and fcc metals $(\mathrm{Al}, \mathrm{Ni}, \mathrm{Cu}, \mathrm{Ag}$, $\mathrm{Au}$, etc. [25]) have also been studied by using DFT.

3.2. Ising Model. Axial next-nearest-neighbor Ising model was firstly applied to describe the polytypic structure of Chou et al. [16]. The analogue spin $S_{i}$ has value 1 or -1 for layer $i$; the sign is determined by the connecting subsequent layer from the close-packed layers. For example, if $i$ layer is A and $i+1$ layer is $\mathrm{B}$, then $S_{i}=1$; if $i$ layer is $\mathrm{A}$ and $i+1$ layer is $\mathrm{C}$, then $S_{i}=-1$. Based on this model, the energy of arbitrary structure can be described by a series expressed as

$$
E=J_{0}-\sum_{n} J_{n} \sum_{i} S_{i} S_{i+n}
$$

where $J_{n}$ is the interaction energy between two layers ( $n=1$ is the first-nearest-neighbor, $n=2$ is the second-nearestneighbor, etc.). $J_{0}$ is the energy where all the interlayer interactions are neglected.

Denteneer and van Haeringen [26] provided the energies of three different structures. For the perfect hcp structure (... ABABAB ...),

$$
E_{\mathrm{hcp}}=J_{0}+J_{1}-J_{2}+O\left(J_{3}\right),
$$

where $\mathrm{O}\left(\mathrm{J}_{3}\right)$ represents the interaction energies between layers with distance over the second-nearest-neighbor. For the perfect fcc structure (...ABCABC ...),

$$
E_{\mathrm{fcc}}=J_{0}-J_{1}-J_{2}+O\left(J_{3}\right) \text {. }
$$


For the double hcp (dhcp) structure (...ABAC ...),

$$
E_{\mathrm{dhcp}}=J_{0}+J_{2}+O\left(J_{3}\right) .
$$

Under this definition and neglecting the series over the second-nearest-neighbor, the energies of the systems of hcp, fcc, and dhcp structures can be calculated by the supercell model. Then the parameters $J_{0}, J_{1}$, and $J_{2}$ can be determined by solving (7) to (9):

$$
\begin{aligned}
& J_{0}=\frac{1}{4}\left(2 E_{\mathrm{dhcp}}+E_{\mathrm{fcc}}+E_{\mathrm{hcp}}\right), \\
& J_{1}=\frac{1}{2}\left(E_{\mathrm{hcp}}-E_{\mathrm{fcc}}\right), \\
& J_{2}=\frac{1}{4}\left(2 E_{\mathrm{dhcp}}-E_{\mathrm{fcc}}-E_{\mathrm{hcp}}\right) .
\end{aligned}
$$

Thus, the energy of arbitrary stacking sequence can be expressed by $J_{n}$.

According to the Ising model, four types of basal plane SFEs of 14 hcp metals were calculated; the results divided hcp metals into three types for the different interlayer interaction features (the results of $J_{n}$ ) [23]. Sandlöbes et al. [20] calculated the SFE of Mg and Mg-Y alloys and found that SFE was decreased by the addition of yttrium. Lu et al. [27] investigated the composition dependence of the SFEs by adding different alloy atoms ( $\mathrm{Mn}, \mathrm{Co}$, and $\mathrm{Nb}$ ) to austenitic stainless steels. They found that Co decreases the SFE, whilst $\mathrm{Nb}$ increases the SFE value. Moreover, Mn decreases the SFE in alloys with less than 16 at. \% Ni; beyond that the SFE rises slightly. Notably, the SFE of Ni was calculated by both the supercell model $\left(127 \mathrm{~mJ} / \mathrm{m}^{2}\right)$ and Ising model $\left(129 \mathrm{~mJ} / \mathrm{m}^{2}\right)$, and both methods agree nicely with experiments $\left(125 \mathrm{~mJ} / \mathrm{m}^{2}\right)$ [25].

3.3. Bond Orientation Model. Chetty and Weinert further analyzed the local environment model and found that the energy also depends on the local environment of the nearestneighbors [17]. Specifically, when considering the local environment of the nearest-neighbors, there are six different energies of a site shown in Table 2. With energies of hcp, fcc, $I_{1}, I_{2}, E$, and $T_{2}$ structures calculated by the supercell model, the results of $\varepsilon_{2}$ to $\varepsilon_{6}$ (listed in Table 2 ) relative to hcp can be determined by

$$
\begin{aligned}
& E_{\mathrm{fcc}}=\varepsilon_{6}, \\
& E_{I_{1}}=2 \varepsilon_{2}+\varepsilon_{4}, \\
& E_{I_{2}}=2 \varepsilon_{2}+2 \varepsilon_{5}, \\
& E_{E}=2 \varepsilon_{2}+2 \varepsilon_{5}+\varepsilon_{6}, \\
& E_{T_{2}}=2 \varepsilon_{2}+\varepsilon_{3}+2 \varepsilon_{4} .
\end{aligned}
$$

The parameters $\varepsilon_{1}$ to $\varepsilon_{6}$ can be calculated by solving (11) and the results are listed in Table 2 .

This model is effectively a second-neighbor model, and the energies of arbitrary fault structures can be described by these parameters. To test the ability of this model in
TABLE 2: The local environment of atoms $i$ and $i \pm 1$; hcp and fcc are atoms of local environment. The parameters $\varepsilon_{1}$ to $\varepsilon_{6}$ denote the energy of atom $i$.

\begin{tabular}{lccc}
\hline$i-1$ & $i$ & $i+1$ & Energy of $i\left(\mathrm{~mJ} / \mathrm{m}^{2}\right)$ \\
\hline hcp & hcp & hcp & $\varepsilon_{1}=0.0$ \\
hcp & hcp & fcc & $\varepsilon_{2}=1.9$ \\
fcc & hcp & fcc & $\varepsilon_{3}=12.5$ \\
hcp & fcc & hcp & $\varepsilon_{4}=17.1$ \\
hcp & fcc & fcc & $\varepsilon_{5}=14.8$ \\
fcc & fcc & fcc & $\varepsilon_{6}=27.1$ \\
\hline
\end{tabular}

predicting the energies of arbitrary structure, two structures were considered. As a first test, the energy of hcp/fcc (111) interface structure denoted by $\mathrm{ABABABABCABC}$ in a 12 atom cell is calculated by both bond orientation model $\left(E=2 \varepsilon_{2}+2 \varepsilon_{5}+4 \varepsilon_{6}=142 \mathrm{~mJ} / \mathrm{m}^{2}\right)$ and supercell model $\left(148 \mathrm{~mJ} / \mathrm{m}^{2}\right)$. The energy of a randomly chosen structure, $\mathrm{ABACBACBCBAC}$, is $E=2 \varepsilon_{2}+2 \varepsilon_{3}+\varepsilon_{4}+4 \varepsilon_{5}+3 \varepsilon_{8}=$ $186 \mathrm{~mJ} / \mathrm{m}^{2}$, while the result calculated by supercell model is $197 \mathrm{~mJ} / \mathrm{m}^{2}$. The results show that the bond orientation model and the supercell model predict very close SFE for Mg.

3.4. Methods for GSFE Calculation. There are two commonly used methods for GSFE calculation: One is computed by direct crystal slip path, in which atoms are only relaxed along the directions perpendicular to the slip planes [28]. The other one is named as climbing image nudged elastic band (CINEB) method, which describes the $\gamma$-surface with precise values of saddle points without constrained way of crystal slips [9].

\section{Calculation of Generalized Stacking Fault Energy}

4.1. Pure Metal. For Mg, the $\gamma$-surface of basal plane along the $(1 / 3)\langle 10 \overline{1} 0\rangle$ direction was listed by Smith [18]. The respective stable SFE from the curve is $36 \mathrm{~mJ} / \mathrm{m}^{2}$. Furthermore, the $\gamma$ surface of four basal plane stacking faults $\left(I_{1}, I_{2}, E\right.$, and $\left.T_{2}\right)$ and others of nonbasal plane stacking faults in the prismatic and pyramidal planes were gained using supercell model. The results show that basal plane stacking fault is the most likely slip direction; the slipping along $\langle 10 \overline{10}\rangle$ could also form a stable stacking fault structure, but its SFE is higher than that of basal plane slip system. Thus, it is difficult to form nonbasal plane stacking faults [28]. The GSF energies of 2layer (AB), 4-layer (dhcp), and 6-layer (ABACAB) structures on basal plane slip were calculated in [22]; the calculated results of unstable SFE for 4-layer and 6-layer structures are higher than that of 2-layer structure. The GSF energies of other hcp metals ( $\mathrm{Be}, \mathrm{Ti}, \mathrm{Zn}$, and $\mathrm{Zr}$ ) were calculated and the $\gamma$-surface of $\{0001\}\langle 11 \overline{2} 0\rangle$ and $\{0001\}\langle 10 \overline{1} 0\rangle$ slip system was shown in Figure 1. The curves illustrate that for different hcp metals the unstable SFE presents at different positions and the values of unstable SFE along $\langle 10 \overline{1} 0\rangle$ are always larger than that along $\langle 11 \overline{2} 0\rangle$ direction. Then, compared by employing DFT within GGA and spin-polarized GGA, results show that the primitive cell volumes influence the GSF energy of 


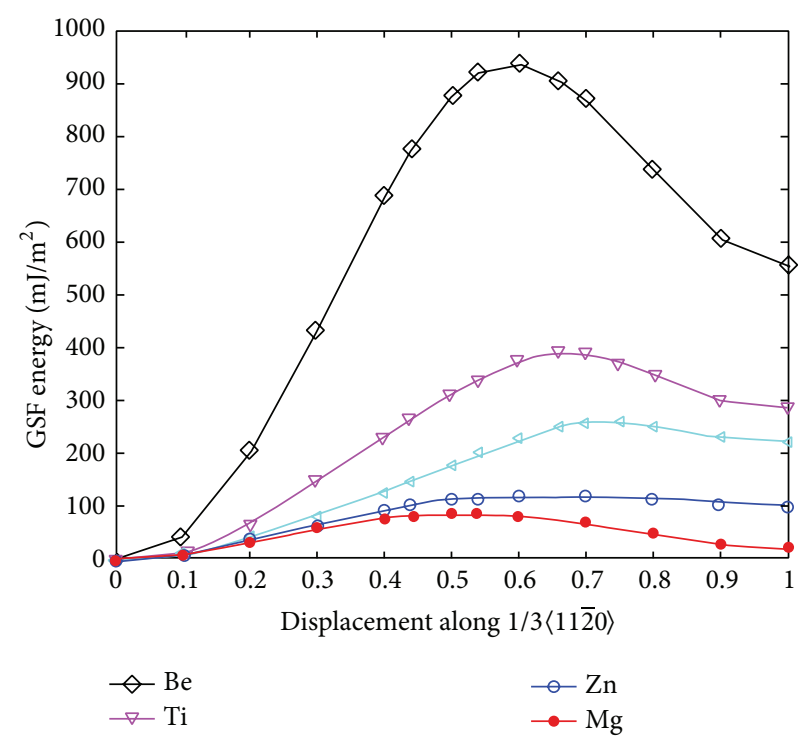

(a)

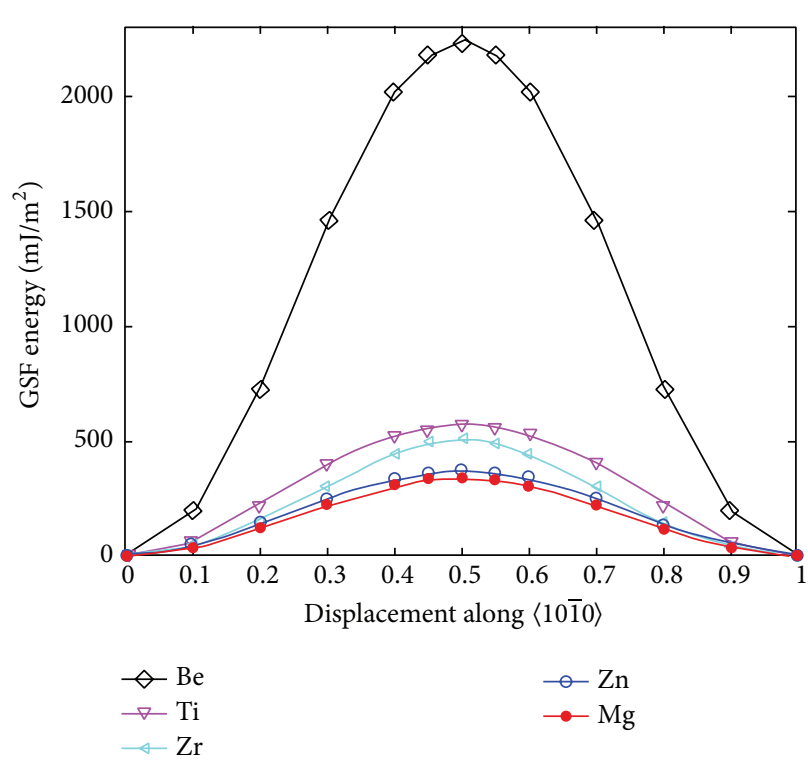

(b)

FIgURE 1: The GSF energy for the basal plane along $\langle 11 \overline{2} 0\rangle$ and $\langle 10 \overline{1} 0\rangle$ directions. The figure is adapted from [24].

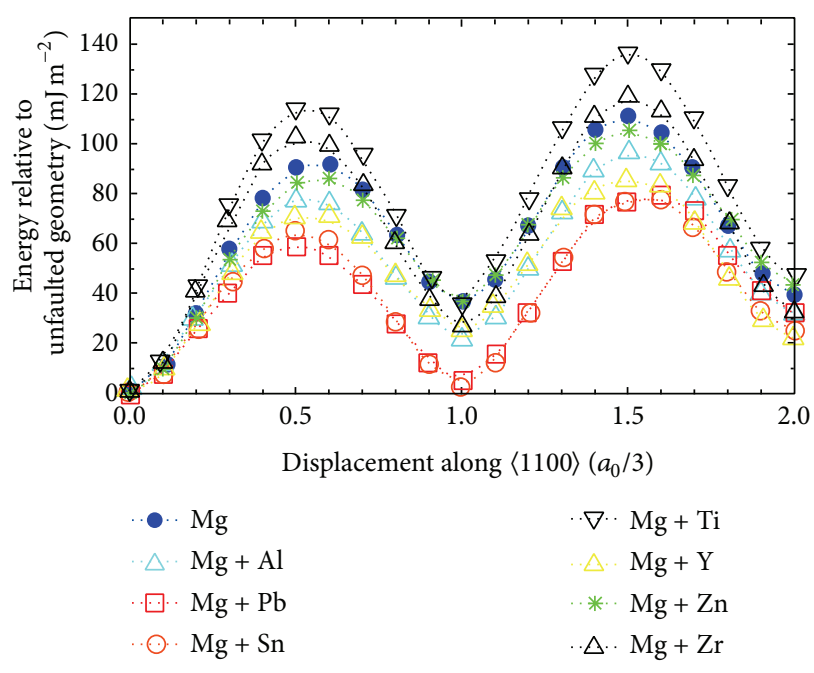

(a)

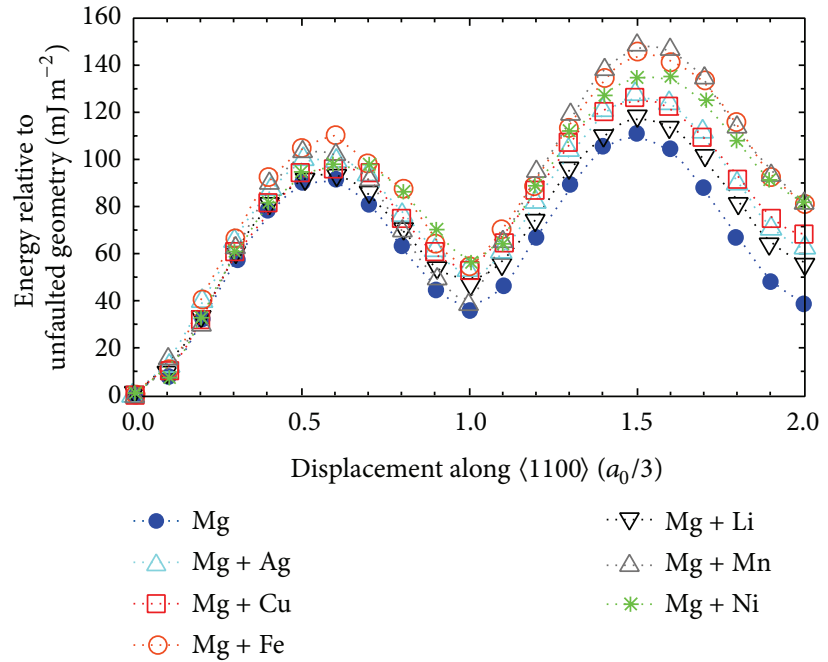

(b)

FIGURE 2: GSF energy curve of Mg and Mg alloys in the (0001)[1100] slip system. The figure is adapted from [29].

hcp metals. For transition metals a spin-polarized calculation should be considered [24].

4.2. Metal Alloys. The GSF energy surface of $\mathrm{Mg}$ alloys has been extensively studied by DFT. Muzyk et al. [29] calculated GSF energy curves of basal plane involving 13 types of metallic alloying elements (Figure 2). They found that the SFE of $\mathrm{Mg}$ reduced obviously when adding $\mathrm{Pb}$ and $\mathrm{Sn}$ atoms. Han et al. [30] considered the effects of alloy atoms on the GSF energy of the basal plane. They concluded that adding $\mathrm{Al}$ atoms tends to decrease the SFEs along the faulting pathways, but adding $\mathrm{Li}$ atoms tends to increase the SFEs along the faulting pathways. Wang et al. [31] considered the GSF energy of a new Mg-Al-Sn alloy of different slip systems. They found that the unstable SFE is reduced due to the doping of $\mathrm{Al}$ and $\mathrm{Sn}$ atoms. Zhang et al. [32] investigated the $\gamma$ surface of $I_{1}$ and $I_{2}$ stacking fault when solute atoms $\mathrm{Y}$ are added. Compared with the $\gamma$-surface of pure $\mathrm{Mg}$, the $I_{1}$ and $I_{2} \gamma$-surfaces of Mg-Y alloy are very similar to that of $\mathrm{Mg}$, as shown in Figure 3. When adding $\mathrm{Y}$ and $\mathrm{Zn}$ simultaneously, the stable and unstable SFEs drastically decrease. The experiment observation of Mg-Y alloys shows the same results. For the LPSO structure, when adding $2 \% \mathrm{Zn}$, the energy of 6-layer structure is compared with that of 2-layer one. The results show that addition of $\mathrm{Zn}$ further stabilizes the 6-layer structure [22]. Another typical hcp metal is Ti. 


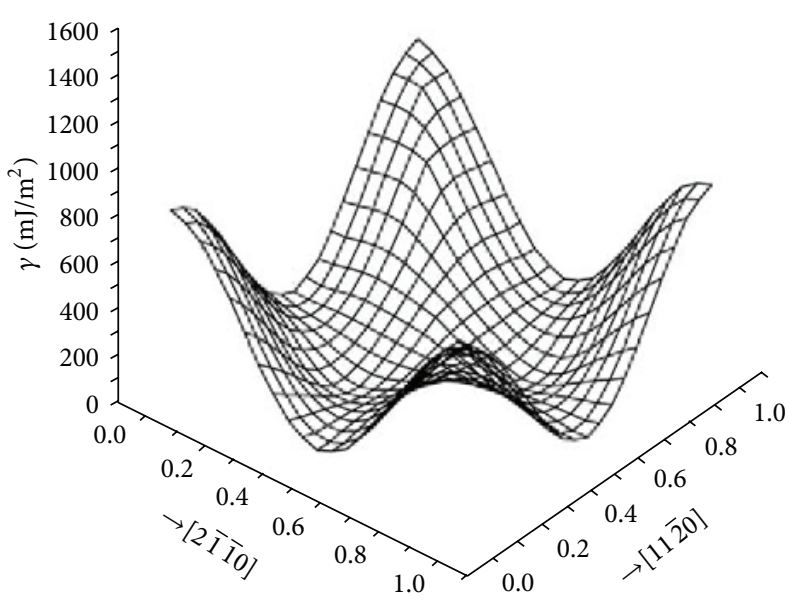

(a)

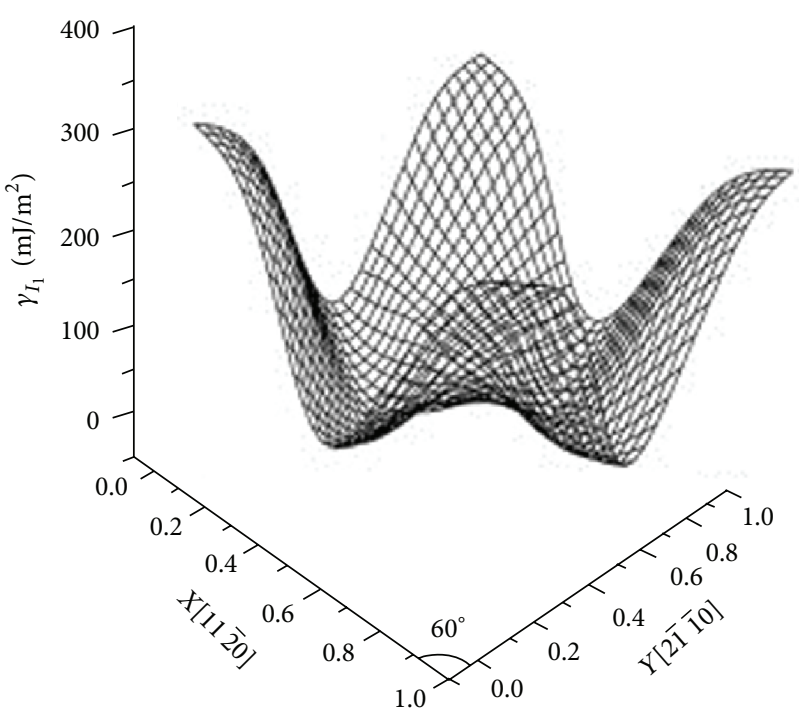

(c)

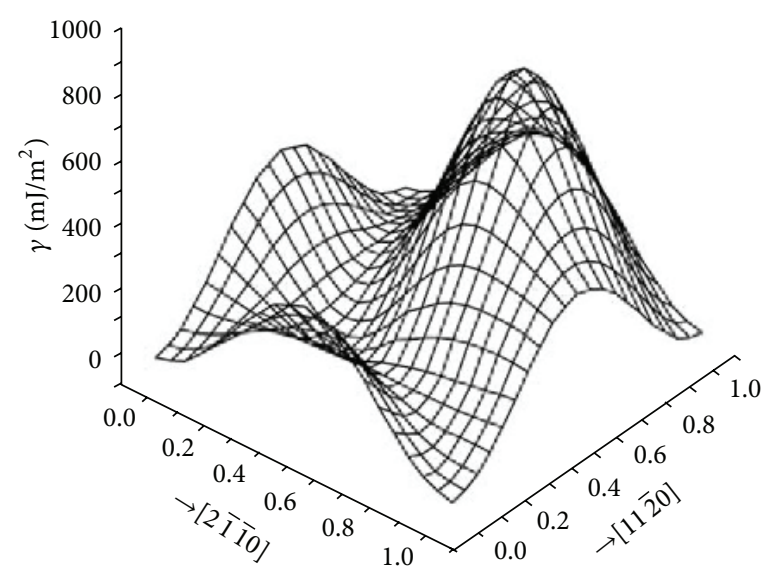

(b)

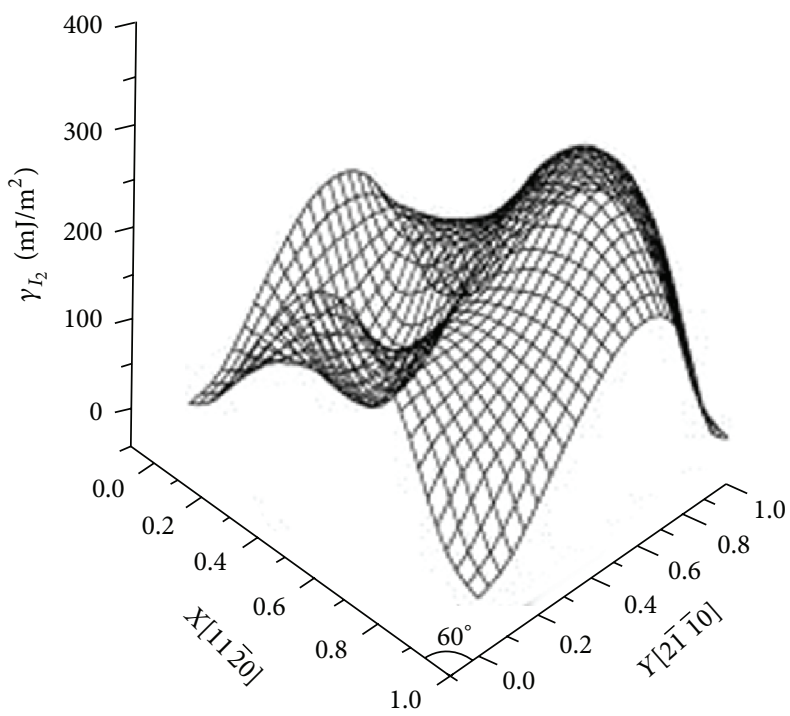

(d)

Figure 3: The $\gamma$-surface of basal plane stacking faults $I_{1}$ of $\mathrm{Mg}(\mathrm{a}), I_{2}$ of $\mathrm{Mg}$ (b), $I_{1}$ of Mg-Y (c), and $I_{2}$ of Mg-Y (d). Plots (a) and (b) are adapted from [28]; plots (c) and (d) are adapted from [32].

Kwasniak et al. [33] investigated the effect of $\mathrm{C}, \mathrm{H}, \mathrm{N}$, and $\mathrm{O}$ on the GSFE of $\alpha$-Ti. They found that solute $\mathrm{H}$ can reduce the SFE considerably, solute $\mathrm{C}$ can improve partial dislocation emission and twin content, and solutes $\mathrm{N}$ and $\mathrm{O}$ can reduce the SFE in considered slip mode [33]. Ghazisaeidi and Trinkle [34] considered the energetics of $\mathrm{O}$ interstitial interactions with a twin boundary and a prismatic stacking fault of $\mathrm{Ti}$, proving that sites located away from the twin boundary are repulsive, while the sites at twin boundary are more attractive to $\mathrm{O}[34]$.

\section{Challenge and Outlook}

In this review, we summarized the theoretical models used for SFE calculations and their applications in metal systems. The available data clearly show that different exchangecorrelation functionals in the framework of DFT can give different results, with large error bars. We thus conclude that it remains difficult to accurately determine the SFE by using the currently used DFT functional. In fact, to accurately describe such systems, some important factors, such as van der Waals forces, many body effects, and dielectric screening effects, should also be carefully considered in DFT calculations. Therefore, the development and application of new DFT that properly include all the above effects will be highly demanded.

\section{Conflict of Interests}

The authors declare that there is no conflict of interests regarding the publication of this paper.

\section{Acknowledgments}

The authors gratefully acknowledge the financial support of Program for New Century Excellent Talents in University 
from Chinese Ministry of Education, National Natural Science Foundation of China (nos. 21403113, 51225102, and 2012CB932203), the Fundamental Research Funds for the Central Universities (no. 30915011330), a project funded by the Priority Academic Program Development of Jiangsu Higher Education Institutions, and the 8th Liuda Rencai Gaofeng (no. B932203) from Jiangsu Province, China.

\section{References}

[1] K. Lu, L. Lu, and S. Suresh, "Strengthening materials by engineering coherent internal boundaries at the nanoscale," Science, vol. 324, no. 5925, pp. 349-352, 2009.

[2] Y. H. Zhao, J. F. Bingert, Y. T. Zhu et al., "Tougher ultrafine grain $\mathrm{Cu}$ via high-angle grain boundaries and low dislocation density," Applied Physics Letters, vol. 92, no. 8, Article ID 081903, 2008.

[3] Y. H. Zhao, Y. T. Zhu, X. Z. Liao, Z. Horita, and T. G. Langdon, "Tailoring stacking fault energy for high ductility and high strength in ultrafine grained $\mathrm{Cu}$ and its alloy," Applied Physics Letters, vol. 89, no. 12, Article ID 121906, 2006.

[4] Y. T. Zhu, X. Z. Liao, and X. L. Wu, "Deformation twinning in nanocrystalline materials," Progress in Materials Science, vol. 57, no. 1, pp. 1-62, 2012.

[5] J. W. Christian and S. Mahajan, "Deformation twinning," Progress in Materials Science, vol. 39, no. 1-2, pp. 1-157, 1995.

[6] Y. T. Zhu, X. Z. Liao, X. L. Wu, and J. Narayan, "Grain size effect on deformation twinning and detwinning," Journal of Materials Science, vol. 48, no. 13, pp. 4467-4475, 2013.

[7] X. Z. Liao, S. G. Srinivasan, Y. H. Zhao et al., "Formation mechanism of wide stacking faults in nanocrystalline Al," Applied Physics Letters, vol. 84, no. 18, pp. 3564-3566, 2004.

[8] X. Z. Liao, Y. H. Zhao, S. G. Srinivasan, Y. T. Zhu, R. Z. Valiev, and D. V. Gunderov, "Deformation twinning in nanocrystalline copper at room temperature and low strain rate," Applied Physics Letters, vol. 84, no. 4, pp. 592-594, 2004.

[9] P. Kwaśniak, P. Śpiewak, H. Garbacz, and K. J. Kurzydłowski, "Plasticity of hexagonal systems: Split slip modes and inverse Peierls relation in $\alpha$-Ti," Physical Review B, vol. 89, no. 14, Article ID 144105, 2014.

[10] S. Allain, J.-P. Chateau, O. Bouaziz, S. Migot, and N. Guelton, "Correlations between the calculated stacking fault energy and the plasticity mechanisms in Fe-Mn-C alloys," Materials Science and Engineering A, vol. 387-389, no. 1-2, pp. 158-162, 2004.

[11] M. Suzuki, T. Kimura, J. Koike, and K. Maruyama, "Strengthening effect of $\mathrm{Zn}$ in heat resistant $\mathrm{Mg}$-Y-Zn solid solution alloys," Scripta Materialia, vol. 48, no. 8, pp. 997-1002, 2003.

[12] Y. Kawamura, K. Hayashi, A. Inoue, and T. Masumoto, "Rapidly solidified powder metallurgy Mg97Zn1Y2 alloys with excellent tensile yield strength above $600 \mathrm{MPa}, "$ Materials Transactions, vol. 42, no. 7, pp. 1172-1176, 2001.

[13] D. Hull and D. J. Bacon, Introduction to Dislocations, Butterworth-Heinemann, Oxford, UK, 2001.

[14] S. L. Shang, W. Y. Wang, B. C. Zhou et al., "Generalized stacking fault energy, ideal strength and twinnability of dilute $\mathrm{Mg}$ based alloys: a first-principles study of shear deformation," Acta Materialia, vol. 67, pp. 168-180, 2014.

[15] V. Vítek, "Intrinsic stacking faults in body-centred cubic crystals," Philosophical Magazine, vol. 18, no. 154, pp. 773-786, 1968.
[16] M. Y. Chou, M. L. Cohen, and S. G. Louie, "Theoretical study of stacking faults in silicon," Physical Review B, vol. 32, no. 12, pp. 7979-7987, 1985.

[17] N. Chetty and M. Weinert, "Stacking faults in magnesium," Physical Review B, vol. 56, no. 17, pp. 10844-10851, 1997.

[18] A. E. Smith, "Surface, interface and stacking fault energies of magnesium from first principles calculations," Surface Science, vol. 601, no. 24, pp. 5762-5765, 2007.

[19] Y. Wang, L.-Q. Chen, Z.-K. Liu, and S. N. Mathaudhu, "Firstprinciples calculations of twin-boundary and stacking-fault energies in magnesium," Scripta Materialia, vol. 62, no. 9, pp. 646-649, 2010.

[20] S. Sandlöbes, M. Friák, S. Zaefferer et al., "The relation between ductility and stacking fault energies in $\mathrm{Mg}$ and $\mathrm{Mg}-\mathrm{Y}$ alloys," Acta Materialia, vol. 60, no. 6-7, pp. 3011-3021, 2012.

[21] Y.-F. Wang, Z.-Z. Wang, N. Yu, X.-Q. Zeng, W.-J. Ding, and B.-Y. Tang, "Microstructure investigation of the $6 \mathrm{H}$-type long-period stacking order phase in $\mathrm{Mg}_{97} \mathrm{Y}_{2} \mathrm{Zn}_{1}$ alloy," Scripta Materialia, vol. 58, no. 10, pp. 807-810, 2008.

[22] T.-W. Fan, B.-Y. Tang, L.-M. Peng, and W.-J. Ding, "Firstprinciples study of long-period stacking ordered-like multistacking fault structures in pure magnesium," Scripta Materialia, vol. 64, no. 10, pp. 942-945, 2011.

[23] Q.-M. Hu and R. Yang, "Basal-plane stacking fault energy of hexagonal close-packed metals based on the Ising model," Acta Materialia, vol. 61, no. 4, pp. 1136-1145, 2013.

[24] X. Wu, R. Wang, and S. Wang, "Generalized-stacking-fault energy and surface properties for HCP metals: a first-principles study," Applied Surface Science, vol. 256, no. 11, pp. 3409-3412, 2010.

[25] M. Chandran and S. K. Sondhi, "First-principle calculation of stacking fault energies in $\mathrm{Ni}$ and Ni-Co alloy," Journal of Applied Physics, vol. 109, no. 10, Article ID 103525, 2011.

[26] P. J. H. Denteneer and W. van Haeringen, "Stacking-fault energies in semiconductors from first-principles calculations," Journal of Physics C. Solid State Physics, vol. 20, no. 32, pp. L883L887, 1987.

[27] S. Lu, Q.-M. Hu, B. Johansson, and L. Vitos, "Stacking fault energies of $\mathrm{Mn}$, Co and $\mathrm{Nb}$ alloyed austenitic stainless steels," Acta Materialia, vol. 59, no. 14, pp. 5728-5734, 2011.

[28] L. Wen, P. Chen, Z.-F. Tong, B.-Y. Tang, L.-M. Peng, and W.J. Ding, "A systematic investigation of stacking faults in magnesium via first-principles calculation," The European Physical Journal B, vol. 72, no. 3, pp. 397-403, 2009.

[29] M. Muzyk, Z. Pakiela, and K. J. Kurzydlowski, "Generalized stacking fault energy in magnesium alloys: density functional theory calculations," Scripta Materialia, vol. 66, no. 5, pp. 219$222,2012$.

[30] J. Han, X. M. Su, Z.-H. Jin, and Y. T. Zhu, "Basal-plane stackingfault energies of Mg: a first-principles study of Li- and Alalloying effects," Scripta Materialia, vol. 64, no. 8, pp. 693-696, 2011.

[31] H.-Y. Wang, N. Zhang, C. Wang, and Q.-C. Jiang, "Firstprinciples study of the generalized stacking fault energy in $\mathrm{Mg}$ 3Al-3Sn alloy," Scripta Materialia, vol. 65, no. 8, pp. 723-726, 2011.

[32] Q. Zhang, L. Fu, T.-W. Fan, B.-Y. Tang, L.-M. Peng, and W.-J. Ding, "Ab initio study of the effect of solute atoms $\mathrm{Zn}$ and $\mathrm{y}$ on stacking faults in Mg solid solution," Physica B: Condensed Matter, vol. 416, pp. 39-44, 2013. 
[33] P. Kwasniak, M. Muzyk, H. Garbacz, and K. J. Kurzydlowski, "Influence of $\mathrm{C}, \mathrm{H}, \mathrm{N}$, and $\mathrm{O}$ interstitial atoms on deformation mechanism in titanium-first principles calculations of generalized stacking fault energy," Materials Letters, vol. 94, pp. 9294, 2013.

[34] M. Ghazisaeidi and D. R. Trinkle, "Interaction of oxygen interstitials with lattice faults in Ti," Acta Materialia, vol. 76, pp. 82-86, 2014.

[35] W. Y. Wang, S. L. Shang, Y. Wang et al., "Effects of alloying elements on stacking fault energies and electronic structures of binary Mg alloys: a first-principles study," Materials Research Letters, vol. 2, no. 1, pp. 29-36, 2014. 

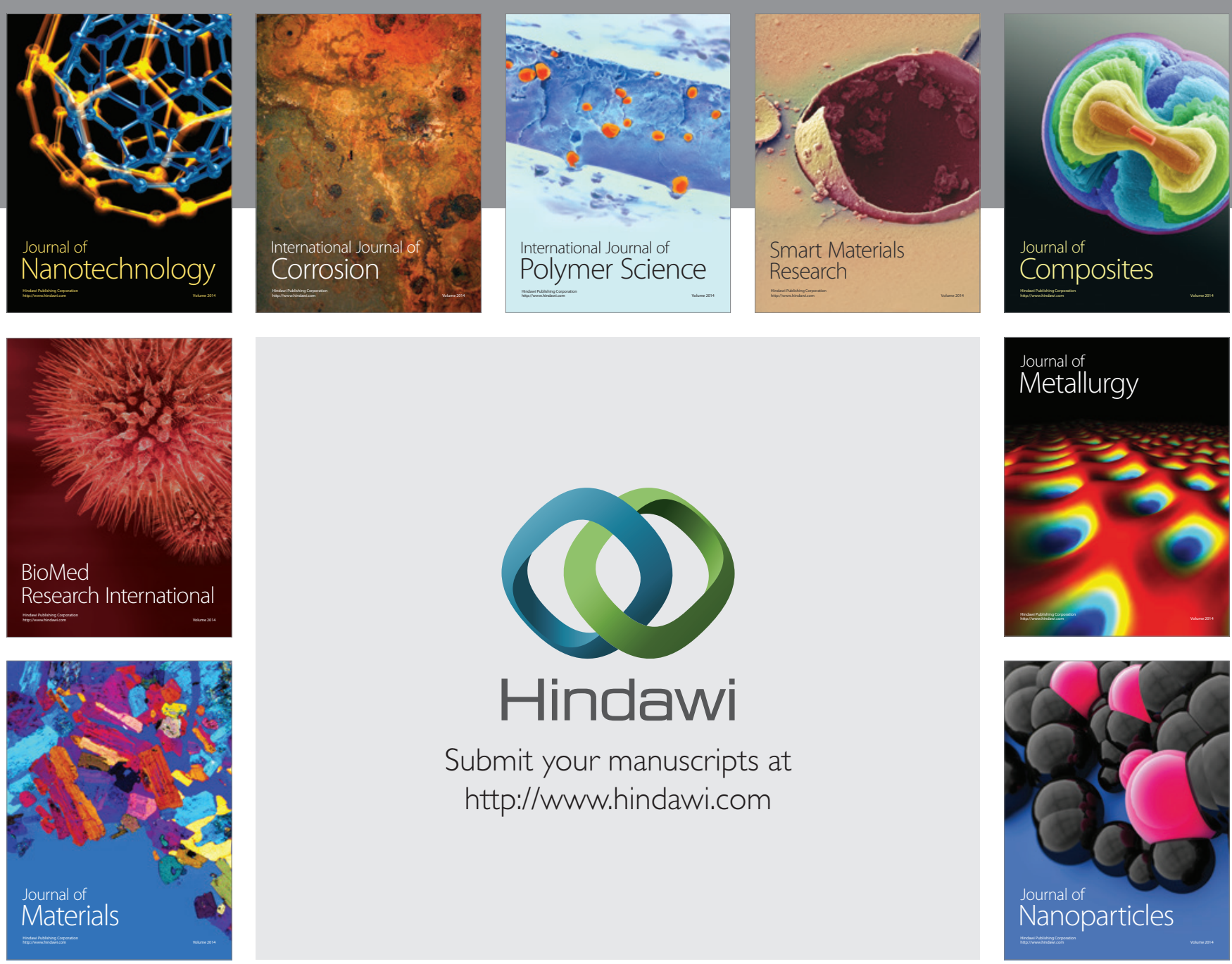

Submit your manuscripts at http://www.hindawi.com
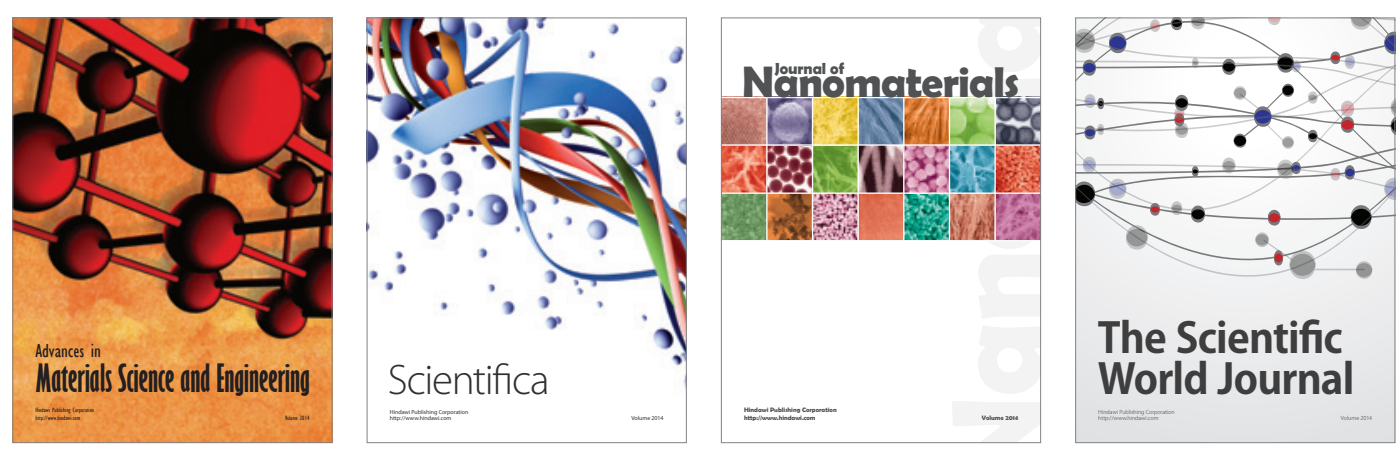

\section{The Scientific World Journal}
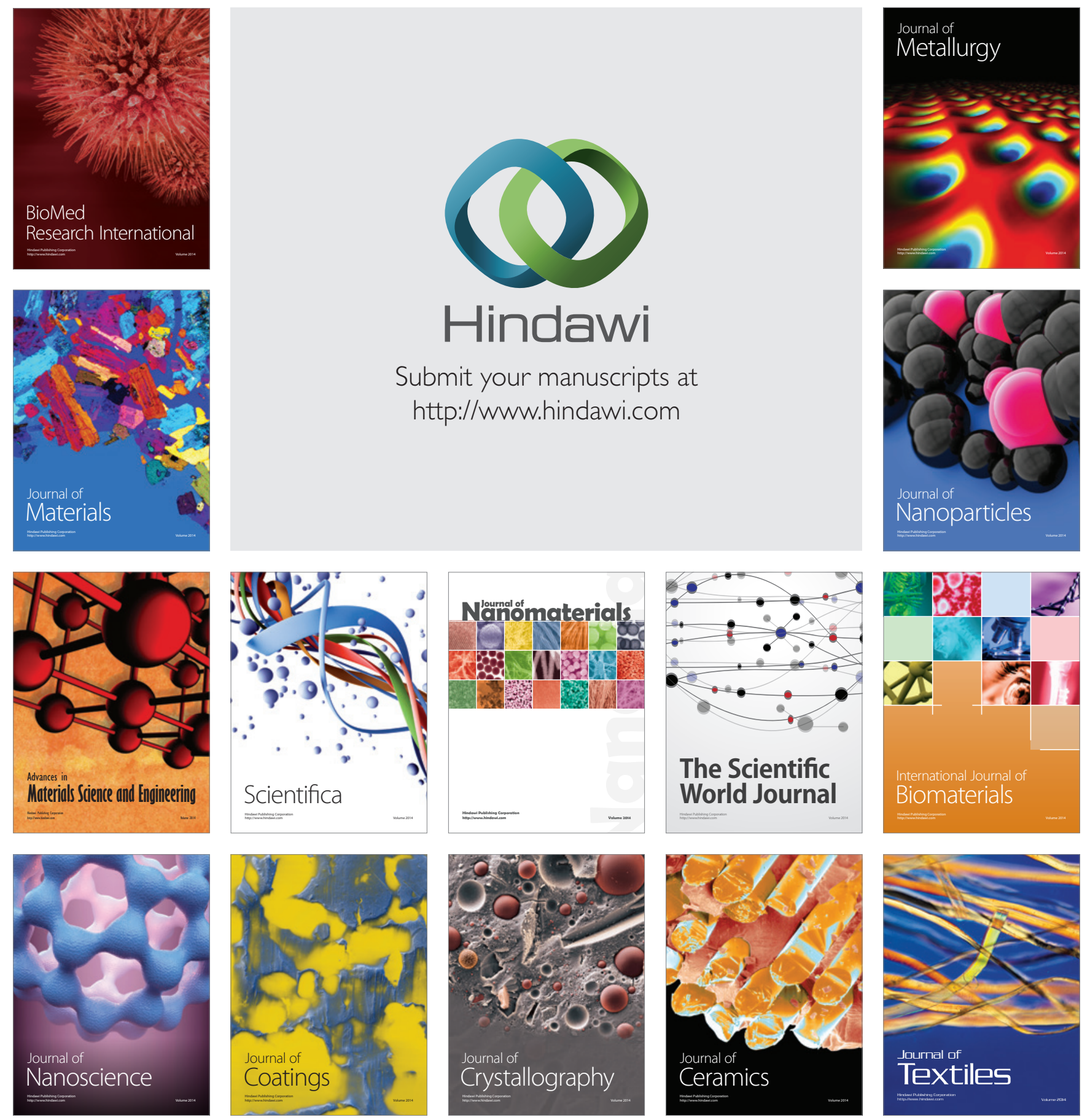